tants de la région, bien connus de l'administration. Un service automobile de la ville assure le transport du lait du point où il a été collecté par les soins du ramasseur à la laiterie municipale dans un délai des plus restreints.

Dans les pays de grande production laitière où l'industrie laitière, fromagère et beurrière fait une grande concurrence au lait destiné à être consommé en nature, des ententes sont toujours possibles entre les Municipalités et les industriels.

C'est ainsi que dans le Calvados, la Mutuelle du Calvados qui réunit tous les fabricants de fromage de ce département et des départements limitrophes s'engage à fournir aux villes, tant d'ailleurs pour la consommation de la population dans son ensemble que pour celle de l'enfance, le lait qui lui est nécessaire.

(A suivre)

\title{
LA SIGNIFICATION ET LA RECHERCHE DU STREPTOCOQUE DANS LE LAIT (1)
}

\author{
Par M. BORNAND
}

(Laboratoire du Service Sanitaire, Lausanne.)

Dans tous les traités d'hygiène alimentaire, les laits provenant de vaches atteintes de mammites à streptocoques sont considérés comme devant être exclus de la consommation. Ces dernières années surtout, cette question relative à la présence du streptocoque dans le lait a pris une certaine importance à la suite d'épidémies d'angines attribuées à la consommation de laits mammiteux.

C'est surtout d'Amérique que nous viennent les principales observations. En 1913, au cours d'une épidémie d'angine survenue à Chicago, transmise par le lait, Rosenau (1) étudie la virulence des streptocoques isolés de la gorge des malades; il constate qu'ils sont virulents pour les animaux, mais qu'ils se différencient du streptocoque pyogènes typique par l'absence de disposition en longues chaînettes. Ce même expérimentateur avec Hess (2) relate en 1917 une autre épidémie frappant 200 personnes avec 11 cas mortels et due à l'ingestion de lait provenant de vaches atteintes de mammittes à streptocoques.

White et Avery (3) en 1913 signalent des faits semblables; l'examen $\mathrm{du}$ troupeau suspect fait constater l'existence d'une inflammation aiguë du pis chez deux vaches; les germes isolés de la gorge des malades présentent les mêmes caractères que ceux provenant du pis des animaux. KuFferath (4) de son côté fait remarquer le danger des streptocoques du lait, car ils peuvent provoquer des entérites mortelles.

(1) Publié dans Traraux de Chimie alimentaire et d'Hyaiène, du Service fédéral suisse. T. XVIII, 1927. 
Le plus grand nombre des cas observés l'ont été en Amérique, mais il est fort probable que si on étudiait chez nous systématiquement l'étiologie d'angines à streptocoques, on trouverait certainement qu'un bon nombre ont le lait pour origine.

Il y a quelques années, un de nos préparateurs ayant examiné un lait mammiteux, le dégusta ; trois jours après cette personne était atteinte d'une sévère angine à streptocoques.

Un grand nombre de recherches ont été faitcs sur les streptocoques du lait, surtout dans le but de déterminer leur pouvoir pathogène, et si des caractères particuliers permettaient de les différencier des vulgaires saprophytes.

C'est avee raison que FouAssien (5) fait remarquer que "lorsque l'on passe en revue tous les travaux entrepris dans ce sens, on est étonné de leur confusion et aussi de leurs conclusions contradictoires ".

Puppel (6) isole des streptocoques du lait de 12 vaches et ne les trouve ni virulents ni hémolytiques; il refuse aux streptocoques du lait un rôle pathogène quelconque. RuHm (7) fait ingérer à des veaux, chevreaux et cobayes, etc. du lait à streptocoques; aucun ne réagit; malgré ces résultats négatifs, l'auteur ne se croit pas autorisé à conclure à l'inocuité d'un lait renfermant des streptocoques; du reste les expériences de Escherich, Holst ont montré qu'un tel lait peut rendre l'homme gravement malade.

Le Sireptococus lacticus qui passe pour un germe saprophyte normal du lait s'est montré pathogène pour le lapin dans les expériences de Hernemann (8). Les lésions étaient identiques à celles produites par Streptococcus pyogenes isolé de l'homme.

Kufferath (9) examinant le lait de 800 vaches en trouve 82 infectées de streptocoques, cet auteur considère que les laits provenant d'animaux atteints de mammite streptococique présentent des dangers pour l'homme. SMrtr et Brown (10) étudient les différences des streptocoques du lait, à la suite d'amygdalite provoquée par cet aliment. Ils ne peuvent affirmer qu'un streptocoque bovin provenant d'une mastite soit l'agent pathogène d'une amygdalite chez l'homme.

Pour Krrchenstern (11), tout lait renfermant des microcoques doit être considéré comme suspect, car ces germes peuvent produire chez la vache une mastite aiguë.

Pour se prononcer sur la pathogénité pour l'homme des streptocoques isolés du lait, les différents expérimentateurs invoquent les caractères morphologiques, culturaux, biologiques (fermentation des sucres, pouvoir hémolytique) ou l'expérimentation sur les animaux.

$\mathrm{Au}$ point de vue morphologique, la dénomination streptocoque telle qu'elle est donnée dans les différents traités, comprend des éléments ovalaires ou sphériques réunis par deux, en chaînettes de 3, 4, 20 éléments et plus; mais la forme classique que l'on rencontre habituellement 
en pathologie humaine et que l'on désigne dans les diagncstics comme streptocoques est celle formée de chaînettes de 4, 5 et surtout (exception faite du pneumocoque) d'un grand nombre d'éléments. En général le streptocoque est extrêmement variable morphologiquement, et la longueur des chaînettes est en relation avec les conditions du milieu. Dans l'organisme, prenons deux pus de même origine, dans l'un, on aura des chaînettes très courtes, dans l'autre, des chapelets de 20, 30 et plus.

Sur les milieux de cultures solides on observe des formes très courtes: dans les milieux liquides bouillon glycosé, on observe toujours de longues chaînettes.

Dans les laits provenant de mastites, on constate des phénomènes semblables, le plus souvent des courtes chaînettes dans le cas de mastites chroniques, et dans les formes aiguës avec beaucoup de pus, de longs chapelets.

Pour ERnst (12), les streptocoques pathogènes du lait sont formés de chaînes de deux cocci, parfois entourés d'une capsule. STAнEL (13) montre qu'il existe 3 formes types du streptocoque de la mammite contagieuse : Une forme longue, une courte et une grèle, qui représentent la même espèce.

J'ai rencontré dans des cas typiques de mammites, surtout chroniques, des formes extrêmement courtes, certaines disposées comme des pneumoeoques, légèrement lancéolées, d'autres disposées en diplocoques mais toutes ces formes ensemencées dans du bouillon glycosé à $1 \%$ ont reproduit après 24 heures d'étuve de très longues chaînettes.

On a invoqué les propriétés biologiques des streptocoques (fermentation des sucres, pouvoir hémolytique) pour différencier les streptocoques pathogènes des saprophytes.

D'après GorinI (14) on rencontre dans le lait trois sortes de streptocoques:

Streptocoques lactiques communs qui se rencontrent comme saprophytes, ils proviennent des matières fécales du bétail, de l'homme; ils répondent à la dénomination de Streptocoques lacticus, type acidifiant.

Streptocoques provenant de la mamelle des vaches considérés comme parasites. Streptocoques mastitidis, type acido-protéolytique.

Streptocoques qui déterminent des affections chez l'homme et chez les bovins, répondant au type Streptocoques pyogenes, type acidoprotéolytique.

Les auteurs américains font jouer un grand rôle au pH des cultures de streptocoques pour la détermination du pouvoir pathogène.

VAN Oyen (15) confirme en partie ces données, et constate que pour $\mathrm{P}_{\mathrm{H}}$ des cultures compris entre 4,56 et 4.77 les streptocoques sont pathogènes pour les bovins et pas pour l'homme; 4,97 à 5.66 pathogènes pour l'homme et dans certaines conditions pour les bovins; 4,86 à 5,42 pathogènes pour le cheval ; 5,14 à 6,28 streptocoques non pathogènes. 
C'est Schottmuller (16) qui étudiant le pouvoir hémolytique des streptocoques, les divise en deux groupes, l'un hémolytique pathogène, l'autre non hémolytique non pathogène pour l'homme. Levaditi (17) expérimentant avec 44 éshantillons de streptocoques isolés de plaies de guerre conclut qu'en comparant l'action sur les sucres et le pouvoir hémolysant et pathogène on peut répartir les streptocoques en deux groupes. Streptocoque du type $S$. pyogenes, hémolysant, pathogène; Streptocoques atypiques non hémolysant et non pathogènes. Un grand nombre d'auteurs ont étudié ces différents caractères, mais on a observé qu'ils ne sont pas constants. En particulier, on a remarqué souvent que lorsque la virulence baisse, l'action sur les hématies ne s'affaiblit pas.

En résumé, les relations qui existent entre le pouvoir fermentatif des sucres, l'hémolyse des globules rouges et la virulence ne sont pas suffisamment constants pour pouvoir prétendre que tel streptocoque isolé de l'organisme humain ou animal est doué de propriétés pathogènes. On a pensé qu'au moyen des réactions d'immunité, on arriverait à résoudre le problème; mais encore de ce côté, la solution n'a pas été trouvéa; dans bon nombre de cas, l'agglutination est positive alors que la fixation du complément ne l'est pas ; on observe aussi de grandes différences en utilisant les sérums agglutinants.

Il reste un deraier point, la virulence; l'épreuve pour l'homme et les animaux de laboratoire est aussi loin d'être concordante. On a remarqué aussi une grande inégalité de virulence entre les streptocoques ; si l'on pense que le streptocoque est l'agent d'une série d'affections, qu'il peut se localiser en différents points de l'organisme en donnant des réactions différentes: Suppurations, endocardites, septicémies, erysipèle, fièvre puerpérale, mastites, etc. on comprend qu'innoculé à l'animal, son pouvoir pathogène ne soit pas constant. Rotschild et ThaLHIMER (18) inoculant des streptocoques d'endocardites obtiennent des arthrites dans $50 \%$ des cas, des endocardites dans $7 \%$ seulement.

Au point de vue de la virulence des différents germes, il est encore des faits qui nous ézhappent; prenons le B. coli hôte normal de l'intestin de l'homme et des animaux; pour des causes que nous ignorons, il peut devenir pathogène : il provoquera des intoxications par les viandes, des cystites, des diarrhées et même de véritables dysenteries ; et pour tant au point de vue morphologique, cultural, biologique ses caractères sont d'une constance absolue. Le Streptocoque, si variable au point de vue de sa forme, de ses différents caractères biologiques, de ses réactions d'immunité pəut à plus forte raison présenter de grandes différences quant à sa virulence. Les streptocoques qu'on rencontre dans le lait présentent des caractères semblables. Vouloir établir une distinction entre streptocoques pathogènes et non pathogènes du lait d'après les caractères que j'ai énumérés, c'est risquer de mettre dans le commerce des laits dangereux pour la santé publique. Du reste, les constata- 
tions faites sur les épidémies provoquées par la consommation de laits provenant de vaches atteintes de mammites sont suffisamment démonstratives pour que l'on écarte du commerce des laits renfermant des streptocoques:

Dans la pratique du laboratoire, l'examen des laits a pour but de dépister la fraude, de déterminer ses qualités nutritives et hygiéniques. Pour que ces examens présentent une valeur, il faut qu'ils soient faits aussi rapidement que possible. Vouloir étudier les caractères biochimiques biologiques ou la virulence de streptocoques isolés de laits pour se prononcer sur leur pathogénité est chose matériellement impossible dans le contrôle journalier, et cela ferait perdre un temps précieux préjudiciable à la santé publique.

D'après Camus (19) il est inutile d'essayer de différencier les espèces pathogènes des non pathogènes, l'inscontance des caractères s'y oppose. Kufferath (20) abonde dans le même sens.

Pratiquement, tout lait renfermant une quantité anormale de leucocytes, présentant des chaînettes de streptocoques, ou des formes par deux ou trois et qui après ensemencement en bouillon glycosé donnent des chaînettes, doit être considéré comme anormal, on suspectera une mammite et le lait sera exclu de la consommation.

J'insiste particulièrement sur le fait que le streptocoque de la mammite contagieuse ne se présente pas nécessairement sous la forme de chaînettes de plusieurs éléments, mais comme je l'ai indiqué au début de ce travail, on rencontre surtout dans les cas chroniques, au début ou à la fin de la maladie des formes par deux parfois trois ou quatre; seul l'ensemencement fera apparaître les longues chaînettes. En ensemençant dans du bouillon glycosé, des dépôts de lait renfermant un grand nombre de diplocoques, mais ne provenant pas de vaches atteintes de mammites infectieuses on n'obtient jamais un développement de formes en chaînettes.

Pour porter le diagnostic de mammite, la méthode de TromsdorF et ses modifications, garde toute sa valeur; l'examen du dépôt coloré par la méthode de GrAM, l'établissement de la formule leucocytaire, l'ensemencement en bouillon glycosé permettront de se prononcer d'une façon certaine, et rapidement.

A côté de l'examen microscopique du sédiment, le chiffre réfractométrique du sérum peut déjà donner des indications si l'on est en présence d'un lait mammiteux; parfois même au début de la maladie, le chiffre est abaissé. J'ai fait à cet effet un certain nombre d'observations que je mentionne ci-dessus avec les résultats de l'examen microscopique et bactériologique; tous les cas ont été confirmés cliniquement.

En résumé, d'après les résultats ci-dessus, on constate qu'un indice de réfraction inférieur à 39 , la présence de nombreux leucocytes, de formes bactériennes en diplocoques, ou en courtes chaînettes qui par 
ensemencement en bouillon glycosé donnent de longs chapelets permettent de suspecter une mammite.

Les caractères morphologiques, biologiques et même la virulence des streptocoques étant inconstants, il n'est pas possible d'établir une distinction entre streptocoques pathogènes et saprophytes.

\begin{tabular}{|c|c|c|c|c|}
\hline$N^{\circ}$ & $\left|\begin{array}{c}\text { Ré- } \\
\text { fract. }\end{array}\right|$ & Leucocytes & Coloré par la méthode de Gram & Culture en bouillon \\
\hline 1 & 37,4 & Nombreux & $\begin{array}{l}\text { Diplocoques, rares chaînettes } \\
\text { de } 3-4 \text { éléments }\end{array}$ & $\begin{array}{l}\text { Nombreuses chaînettes de } 10 \\
\text { à } 20 \text { éléments }\end{array}$ \\
\hline 2 & 38,8 & Très nombreux & Diplocoques, rares chaînettes & Nombreuses \\
\hline 3 & 34,6 & Pus & $\begin{array}{l}\text { Quelques chaînettes de 4-5 } \\
\text { éléments }\end{array}$ & $\begin{array}{l}\text { Chaînettes nombreuses et très } \\
\text { longues }\end{array}$ \\
\hline 4 & 37,3 & Nombreux & Chaînettes de $3-4$ éléments & $\begin{array}{l}\text { Nombreuses chaînettes de } 10 \\
\text { à } 15 \text { éléments }\end{array}$ \\
\hline 5 & 38,2 & id. & $\begin{array}{l}\text { Pas de chaînettes, formes par } \\
\text { deux }\end{array}$ & $\begin{array}{l}\text { Nombreuses chaînettes lon- } \\
\text { gues }\end{array}$ \\
\hline 6 & 32,5 & Pus & ttes de $3-4$ éléments & Chaînettes longues \\
\hline 7 & 37,0 & Nombreux & Diplocoques & id. \\
\hline 8 & 37.8 & Pus & - & - \\
\hline 9 & 38,2 & Nombreux & $\begin{array}{l}\text { Nombreux diplocoques, châ̂- } \\
\text { nettes de } 3.4 \text { éléments }\end{array}$ & Très longues chaînettes \\
\hline 10 & 37 & id. & $\begin{array}{l}\text { Rares ehaînettes de } 3-4 \text { élé- } \\
\text { ments }\end{array}$ & 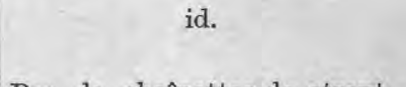 \\
\hline 11 & 39,0 & Normal & Nombreux diplocoques & Pas de chaînettes de strept. \\
\hline 12 & 39,0 & id. & & id. $=$ \\
\hline 13 & 38.5 & Nombreux & $\begin{array}{l}\text { Nombreux diplocoques rares } \\
\text { formes en chaînettes }\end{array}$ & Très nombreuses chaînettes \\
\hline 14 & 38,5 & id. & Diplocoques & Nombreuses chaînettes \\
\hline 15 & 37,7 & Pus & $\begin{array}{l}\text { ix diplocoques, quel- } \\
\text { haînettes }\end{array}$ & Très nombreuses chaînettes \\
\hline 16 & 37.2 & $T-100=$ & Diplocoques nombreux & $\begin{array}{l}\text { Nombreuses chaînettes de } 15 \\
\text { á } 20 \text { éléments }\end{array}$ \\
\hline 17 & 36.2 & Très nombreux & $\begin{array}{l}\text { Quelques ehaînettes de } 3-4 \\
\text { éléments }\end{array}$ & $\begin{array}{l}\text { Nombreuses chaînettes lon. } \\
\text { gues }\end{array}$ \\
\hline 18 & 35,5 & id. & id. & id. \\
\hline 19 & 39.6 & Peu & Diplocoques - & Pas de chaînettes \\
\hline 20 & 37 & Nombreux & Diplocoques nombreux & $\begin{array}{l}\text { Grand nombre de chaînettes } \\
\text { longues }\end{array}$ \\
\hline 21 & 38.5 & Nombreux sang & $\begin{array}{l}\text { Diplocoques, quelques formes } \\
\text { par } 3 \text { et } 4\end{array}$ & $\begin{array}{l}\text { Nombreuses chainettes de } 15 \\
\text { à } 20 \text { éléments }\end{array}$ \\
\hline 22 & 38,3 & id. & id. & id. \\
\hline 23 & 39,0 & Normal & Diplocoques nombreux & Pas de chainettes \\
\hline 24 & 39 & id. & Quelques diplocoques & id. \\
\hline 25 & 39 & id. & id & \\
\hline
\end{tabular}


Au point de vue de la santé publique, il est nécessaire de porter le diagnostic de mammite aussi rapidement que possible, et exclure de la consommation tout lait renfermant une quantité anormale de leucocytes, des streptocoques, quelle que soit l'origine de ces derniers.

\title{
BIBLIOGRAPHIE
}

[1] Rosenau. Ref. Bull. de l'Institut Pasteur, 1913, p. 149.

[2] Rosenau et Hess, Bull. de l'Institut Pasteur, 1917, p. 559.

[3] White et Avery. Bull. de l'Office Intern. d'Hygiène publique, 1914, p. 548.

[4] Kufferath. Annales Pasteur, 1921, p. 170.

[5] Fouassier. $7^{\text {e }}$ Congrès international de Laiterie. Paris, 1926, p. 193.

[6] PUPpex, Ztschr. f. Hyg., T. 70, p. 449.

[7] Ruнm. Rev. Gén. Méd. Vét., 1909. T. 13. p. 468.

[8] Heinemann. Ref. Bull. Pasteur, 1907, p. 146.

[9] Travail cité.

[10] Sмiтt et Brown. Bull. Pasteur, 1915, p. 340.

[11] Kirchenstein. Trav. de Chim. Alim. et d'Hyg., 1917, p. 145.

[12] Ernst. Ref. Rev. Gén. de Méd. Vet., 1910, p. 203.

[13] Staheli. Rev. Gén. de Méd. Vét,, 1906, p. 345.

[14] Gorini. 7e Congrès international de Laiterie, Paris, 1926, p. 197.

[15] VAN OYen. Le Lait, 1923, p. 813.

[16] Schotтmüllek. Münchn. Med. Wochenschr., 1903, № 20.

[17] Levaditi. C. R. Soc. Biologie, 1918, p. 406.

[18] Rotschild et Thalmimer. Cité par Brunet et Weissenbach. Bull. Pasteur. 1918. p. 629.

[19] Camus. Thèse Lyon.

[20] Travail cité.

\section{LES RELATIONS ENTRE L'ACIDITÉ ACTUELLE ET L'ACIDITÉ POTENTIELLE DU LAIT}

\author{
par M. A. TAPERNOUX
}

Chei des travaux de Chimie à l'Ecole Vétérinaire de Lyon (Suite)

Le lait qui a subi cette altération devient d'abord aigre et inconsommable (acidité supérieure à $24^{\circ} \mathrm{D}$ ); il est difficile ou impossible à utiliser soit pour la fabrication des fromages, soit pour la préparation des dérivés du lait, ce qui explique tout l'intérêt que portent les industriels laitiers à la question de l'acidité.

L'acidité continuant à augmenter, le lait qui d'abord ne coagulait pas à l'ébullition finit par cailler quand on le porte à haute température, puis il caille spontanément même à la température ordinaire. On admet que le lait coagule à l'ébullition dès que son acidité de titration dépasse $25^{\circ} \mathrm{D}$. et qu'il coagule spontanément pour une acidité de $80^{\circ} \mathrm{D}(1)$.

(1) G, Hinard. Analyse des laits, p. 54. 